\title{
Effect of Calcaneal Taping on Peak Plantar Pressure of Forefoot and Rearfoot during Gait
}

\author{
Jong-Hyuck Weon', Goen-Su Kim², Do-Young Jung ${ }^{3}$ \\ ${ }^{1}$ Physiopathologic Science Institue, Department of Physical Therapy, Joongbu University; ${ }^{2}$ Multi-Care Specialization Project, Joongbu University; \\ ${ }^{3}$ Physiopathologic Science Institute, Department of Physical Therapy, Joongbu University, Geumsan-gun, Chungnam, Korea
}

Purpose: The purpose of this study was to determine the effects of calcaneal taping on peak plantar pressure of rearfoot and forefoot while walking.

Methods: Fifteen healthy subjects with normal feet participated in this study. Inclusion criteria were as follows: (1) no disturbance of gait and foot pain, (2) normal range of motion of ankle joint, (3) no foot deformity. Pedoscan was used for recording of plantar pressure data during walking. The participants walked along a 12-m walkway before and after application of calcaneal taping. The plantar pressure gait was measured 3 times under barefoot and calcaneal taping conditions randomly at a speed practiced with the metronome during gait. The peak plantar pressure data were calculated for medial and lateral areas of the rearfoot and forefoot. The paired t-test was used to determine significant differences in peak plantar pressure of rearfoot and forefoot before and after application of calcaneal taping. A p-value less than 0.05 was accepted as significant.

Results: The calcaneal taping resulted in statistically significant decreases in peak plantar pressure of the rearfoot (medial side: $p=0.03$; lateral side: $p=0.01$ ). However, there were no significant changes in peak plantar pressure of the forefoot (medial side: $p=0.45$; lateral side: $p=0.40$ ).

Conclusion: The calcaneal taping is recommended to reduce plantar pressure of the rearfoot in weight-bearing activities in subjects with plantar heel pain caused by atrophy of the fat pad.

Keywords: Calcaneal taping, Plantar heel pain, Plantar pressure

\section{INTRODUCTION}

Plantar heel pain is a common symptom that occurs in $15 \%$ of adult patients with foot disease. It is caused by a variety of diseases including plantar fasciitis, heel spur, calcaneal fracture, rupture of plantar fascia, planar nerve entrapment, and heel pad syndrome.

Plantar fasciitis is the most common foot disease cause of plantar heel pain. It occurs most commonly in the 40 to 60 age group. It is associated with various factors including limited dorsiflexion of the ankle joint excessive pronation and supination, prolonged weight bearing activities such as standing and walking. ${ }^{2-4}$

Although the pathological mechanism of plantar fasciitis is unknown clearly, the result of the biopsy study showed that secondary

Received Dec 13, 2015 Revised Dec 18, 2015

Accepted Dec 21, 2015

Corresponding author Do-Young Jung

E-mail ptsports@joongbu.ac.kr degenerative change occurred without acute inflammation in with patients with strain or minimal damage of plantar fascia. ${ }^{5} \mathrm{Heel} \mathrm{pad}$ syndrome is sometimes misdiagnosed as plantar fasciitis.

Heel pad syndrome involves deep pain of the planter heel when walking with bare feet or on hard ground. It is caused by injury and fat atrophy as well as inflammation of the heel pad. ${ }^{1}$ Atrophy of the heel fat pad is accompanied by loss of water, collagen and elastic tissue after 40 s ages. ${ }^{3}$ The heel pad is composed of fibrous septa and closely packed fat cells. Fibrous septa, composed of rich collagen and elastin fibers, prevent deforming or leaking of adipose chambers in a closely-packed compartment. ${ }^{6}$

The heel pad effectively absorbs shock and damping vibration during activities of weight bearing. ${ }^{7}$ Part of the impact energy

Copylight (C2015 The Korea Society of Physical Therapy

This is an Open Access article distribute under the terms of the Creative Commons Attribution Non-commercial License (Http:// creativecommons.org/license/by-nc/3.o.) which permits unrestricted non-commercial use, distribution, and reproduction in any medium, provided the original work is properly cited. 
caused in the deformation of the heel pad during ambulation is dissipated as heat, and part is restored to the subsequent elastic energy. Atrophy of fat pad or rupture of fibrous septa is present in aging heel pads. ${ }^{8}$

Under these conditions, overload of soft tissue surrounding calcaneus causes inflammation accompanied by pain and edema in the plantar heel during sustained weight bearing. Therefore, stiffness and elasticity of the heel fat pad are very important in cushioning, because of decrease of 20\%-25\% of total contact force during heel strike. Previous studies have reported that feet with heel pad syndrome were stiffer than feet in the control group. ${ }^{9,10}$ Also, previous studies determined elasticity of the heel pad in patients with plantar heel pain and elderly people. ${ }^{11-13}$ Ozdemir et al. ${ }^{12}$ reported that elasticity of the heel pad was reduced with increasing age and weight in 50 subjects with plantar heel pain. Hsu et al. ${ }^{11}$ found that the compressibility index (CI: ratio of thickness of the loaded heel pad to it unloaded) and energy dispersion ratio were greater in elderly subjects compared to younger subjects.

Various methods including resting, ice pack therapy, anti-inflammatory drug, painkiller, stretching of calf muscles, heel cup, taping, corrective footwear, and orthosis were used in management of plantar heel pain in clinic or sports fields. Most previous studies evaluated low-dye taping and calcaneal taping for management of plantar fasciitis caused by excessive pronation of the foot. Noran and Kennedy ${ }^{14}$ reported that low-dye taping significantly increased the pressure of lateral midfoot and decreased the pressure of medial forefoot and rearfoot. Hyland et al. ${ }^{15}$ reported that calcaneal taping was more effective in reducing plantar heel pain than sham taping and stretching of plantar fascia. Although some research determined effectiveness of taping to control pronation of the foot on the change of foot pressure, no study to evaluate taping to reduce the load of heel pad through measuring plantar pressure has been reported.

The purpose of this study is to determine the effect of calcaneal taping for reducing load of the heel pad on plantar pressure during gait and to introduce a useful taping method for patients with plantar heel pain caused by atrophy of fat pad or loss of elasticity.

This study established a hypothesis that the peak plantar pressures of medial and lateral rearfoot with application of calcaneal taping on the heel pad would be lower than those in barefoot condition during gait.

\section{METHODS}

\section{Subject}

Fifteen normal subjects participated in this study. Inclusion criteria were as follows: (1) no disturbance of gait and foot pain, (2) normal range of motion of ankle joint, (3) 2 degree in varus positon $\leq$ resting calcaneal stance position (RCSP) angle $\geq 2$ degree in valgus position, (4) no foot deformity such as hallux valgus or claw toe. Each participant's consent was voluntarily established after a full explanation of the experiment. General characteristics of participants were $26 \pm 4.46$ years of age, $169.80 \pm 7.49 \mathrm{~cm}$ in height, and $64.73 \pm 12.37$ $\mathrm{kg}$ in body weight.

\section{Method}

\section{1) Experimental equipment}

A pressure sensor of mat type (Pedoscan, DIERS Inc., Germany) was used for measurement of pressure of the foot according to conditions of barefoot or calcaneal taping. The mat was composed of a 4,096 pressure sensor in Pedoscan, was $50 \mathrm{~cm}$ in width and length, respectively. The sampling rate was $300 \mathrm{~Hz}$ of frequency. ${ }^{16,17}$

Heel taping for reducing the weight of the heel pad was used with non-elastic white tape, $4 \mathrm{~cm}$ in width (PerformPlus, Muller Sports Medicine Inc., Wisconsin, USA).

\section{2) Experimental procedure}

Pedoscan's mat, composed of a pressure sensor, was fixed in the middle of a passageway of $10 \mathrm{~m}$-length. To maintain a constant gait velocity, average gait velocity was calculated by walking at a self-selected speed by 3 times repetition. The calculated gait speed and metronome speed matched in each subject. Then, the participants practiced pressing the middle of the mat during gait, with each subject maintaining the same step length. Plantar pressure during gait was measured 3 times under barefoot and calcaneal taping conditions randomly at a speed practiced with the metronome.

For application of calcaneal taping, the tested foot was placed comfortably on a towel on the edge of the bed in the supine position. An anchor tape was applied from the lateral border of the heel at the calcaneocuboid joint to the medial end of the heel at the talonavicular joint (Figure 1A). The tape was applied to the lateral border of the sole and then stretched tight using one hand, while the fat pad in the medial and lateral border of the heel was gathered in the 

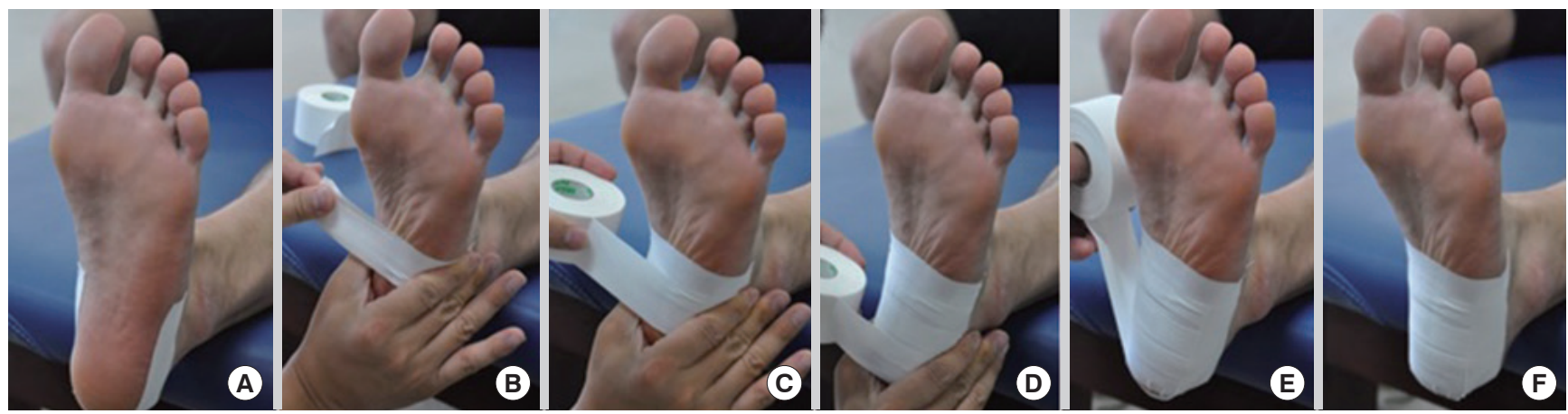

Figure 1. Application of calcaneal taping.

Table 1. Peak plantar pressures of rear- and forefoot in two conditions during gait

(Unit: N/cm²)

\begin{tabular}{|c|c|c|c|c|c|c|}
\hline \multirow{2}{*}{ Foot part } & \multirow{2}{*}{ Side } & \multicolumn{2}{|c|}{ Conditions } & \multirow{2}{*}{ Differences } & \multirow{2}{*}{$\mathrm{t}$} & \multirow{2}{*}{$p$} \\
\hline & & Barefoot & Calcaneal taping & & & \\
\hline \multirow[t]{2}{*}{ Rearfoot } & Medial & $15.21 \pm 1.40$ & $13.89 \pm 1.41$ & $1.3 \pm 1.46$ & 3.52 & 0.03 \\
\hline & Lateral & $14.00 \pm 0.97$ & $13.16 \pm 1.33$ & $0.8 \pm 1.14$ & 2.86 & 0.01 \\
\hline \multirow[t]{2}{*}{ Forefoot } & Medial & $12.15 \pm 1.64$ & $11.80 \pm 1.88$ & $0.35 \pm 1.72$ & 0.79 & 0.45 \\
\hline & Lateral & $10.94 \pm 2.30$ & $11.28 \pm 1.53$ & $-0.34 \pm 1.53$ & -0.87 & 0.40 \\
\hline
\end{tabular}

center by the other hand. The tape was applied from lateral to medial direction of the heel pad (Figure 1B). Second tape was applied repeatedly placing the first tape close together and half overlapping (Figure 1C). The third or fourth tape can be applied according to the subject's foot size (Figure 1D). Another anchor tape was applied to fix the small tapes as the first anchor. Proper adherence of tape was confirmed.

\section{3) Data analysis and statistical analysis}

The date of pressure distribution was measured by peak plantar pressure during gait using the DICAM program of Pedoscan. Peak plantar pressure was measured by distinguishing each area between medial and lateral areas in forefoot and hindfoot. The paired t-test was performed for comparison of the peak plantar pressure on medial and lateral areas in forefoot and rarefoot according to barefoot and calcaneal taping. A p-value less than 0.05 was accepted as significant. Statistical analyses were performed using SPSS 12.0 for windows (SPSS Inc., Chicago, IL, USA).

\section{RESULTS}

The mean of peak plantar pressures in forefoot and rearfoot according to barefoot and calcaneal taping conditions are shown in Table 1. Calcaneal taping resulted in statistically significant decreases in peak plantar pressure of rearfoot (medial side: $\mathrm{p}=0.03$; lateral side: $\mathrm{p}=0.01)$. However, there were no significant changes in peak plantar pressure of the forefoot (medial side: $\mathrm{p}=0.45$; lateral side: $\mathrm{p}=0.40)$.

\section{DISCUSSION}

The results of this study showed that calcaneal taping significantly reduced the peak plantar pressure on medial and lateral areas of rearfoot. However, there were no significant changes in peak plantar pressure of the forefoot.

Previous studies evaluated change in foot pressure with application of taping, orthosis, and various shoe inserts to subjects with plantar heel pain such as plantar fasciitis. Russo and Chipchase ${ }^{18}$ found that in application of low-dye taping to subjects with a normal foot, the peak plantar pressure of medial and lateral areas in hindfoot increased (medial area: mean difference $=3.7 \mathrm{~N} / \mathrm{cm}^{2}$; lateral area: mean difference $=4.0 \mathrm{~N} / \mathrm{cm}^{2}$ ), but medial and lateral forefoot decreased (medial area: mean difference $=-8.3 \mathrm{~N} / \mathrm{cm}^{2}$; lateral area: mean difference $=-2.6 \mathrm{~N} / \mathrm{cm}^{2}$ ). Contrary to previous study, in this study the peak plantar pressure of rearfoot decreased with application of calcaneal taping. Previous researchers speculated that the peak plantar pressure of the midfoot would be moved to the lateral direction because low-dye taping decreased motion of eversion, 
thus causing change in plantar pressure of the lateral rarefoot. Also, we indicated that the peak plantar pressure of rearfoot decreased, because the fat pad around heel may be moved to sole of foot by the calcaneal taping.

In this study calcaneal taping was applied from lateral to medial direction to prevent pronation of rearfoot. However, we insist that peak plantar pressure of rearfoot could be decreased because calcaneal taping was applied to gather the fat pad of the medial and lateral border to the middle of the plantar heel. Therefore, calcaneal taping is recommended for patients with plantar heel pain due to atrophy of the fat pad as well as plantar fasciitis related to excessive pronation of rearfoot.

Based on previous studies and literature, a modified method, from a modified taping method introduced by Maria and Mark, ${ }^{19}$ was applied in this study. Their taping method could unload the pressure of the heel fat pad, by applying the tape from proximal to distal of the foot during gathering of the fat pad in the middle of the plantar heel. On the other hand, in this study we applied the tape from distal to proximal of foot to prevent movement of the fat pad to forefoot (Figure 1B-1D). In a control randomized trial Hyland et al. ${ }^{15}$ reported that calcaneal taping decreased pain more and improved the functional activities more than stretching of plantar fascia during a period of 1-week.

Calcaneal taping applied in previous study could not gather the fat pad, it was just applied from medial to lateral direction to prevent excessive pronation of foot. Plantar heel pain is caused by microdamage due to repetitive and high stress of heel pad in sustained weight bearing activities. Antalgtic gait was shown to decrease the loading of planter heel in patient with plantar heel pain. ${ }^{20-22}$ Sullivan et al. ${ }^{21}$ reported that the peak plantar pressure of the heel in the experimental group with plantar heel pain was less than in the control group with normal foot They also found that as the pain was increased, the lower the peak plantar pressure of the heel. The load of forefoot is increased as a strategy to decrease the pain in patients with plantar heel pain. Therefore, the change of foot pressure and antalgic gait with application of calcaneal taping in patients with plantar heel pain will be determined in further study. In this study, calcaneal taping was applied from lateral to medial direction, in other words, taping was applied towards inversion, which prevented pronation of the foot in gait. Similar to the result of research by $\mathrm{Vi}$ cenzino et al. ${ }^{23}$ there was no significant difference in change of peak plantar pressure in forefoot, but that of medial and lateral areas in forefoot decreased and increased, respectively. Although plantar pressure in the midfoot was not measured, we speculate that calcaneal taping may affect peak plantar pressure more in the midfoot than in the forefoot.

There are some limitations of this study. First the gait speed in each condition or trial is unknown. The subject's walking speed was maintained using a metronome because it could affect change of foot pressure. Second, the results of this study are difficult to generalize to all adults. Atrophy of fat pad occurs mainly in adults older than 40 years old, but the average age of subjects in this study was 26 years old (range: 23-40 years old), thus it cannot represent all normal adults. Finally, we did not know whether the results for change of foot pressure in patients with plantar heel pain are the same as those in normal feet. Finally, in this study taping was applied to normal subjects, thus we did not know whether or not it causes change of foot pressure in patients with pain in the heel pad. Therefore, the long-term effect of calcalneal taping on change of foot pressure and pain in subjects with plantar heel pain will need to be determined.

The results of this study showed that calcaneal taping significantly reduced the peak plantar pressure on medial and lateral areas of rearfoot. These results indicate that calcaneal taping could be effective in decreasing pain of heel in weight-bearing activities in patients with plantar heel pain caused by atrophy of the fat pad.

\section{ACKNOWLEDGEMENTS}

This paper was supported by Joongbu University Research \& Development Fund, in 2015.

\section{REFERENCES}

1. Tu P, Bytomski JR. Diagnosis of heel pain. Am Fam Physician. 2011; 84(8):909-16.

2. Buchbinder R. Clinical practice. Plantar fasciitis. N Engl J Med. 2004; 350(21):2159-66.

3. Neufeld SK, Cerrato R. Plantar fasciitis: Evaluation and treatment. J Am Acad Orthop Surg. 2008;16(6):338-46.

4. Riddle DL, Pulisic M, Pidcoe P et al. Risk factors for plantar fasciitis: A matched case-control study. J Bone Joint Surg Am. 2003;85-A(5):872-7.

5. Lemont H, Ammirati KM, Usen N. Plantar fasciitis: A degenerative process (fasciosis) without inflammation. J Am Podiatr Med Assoc. 2003; 93(3):234-7.

6. Prichasuk S. The heel pad in plantar heel pain. J Bone Joint Surg Br. 
1994;76(1):140-2.

7. Bennett MB, Ker RF. The mechanical properties of the human subcalcaneal fat pad in compression. J Anat. 1990;171:131-8.

8. Paul IL, Munro MB, Abernethy PJ et al. Musculo-skeletal shock absorption: Relative contribution of bone and soft tissues at various frequencies. J Biomech. 1978;11(5):237-9.

9. Rome K, Webb P, Unsworth A et al. Heel pad stiffness in runners with plantar heel pain. Clin Biomech (Bristol, Avon). 2001;16(10):901-5.

10. Tsai WC, Wang CL, Hsu TC et al. The mechanical properties of the heel pad in unilateral plantar heel pain syndrome. Foot Ankle Int. 1999; 20(10):663-8.

11. Hsu TC, Wang CL, Tsai WC et al. Comparison of the mechanical properties of the heel pad between young and elderly adults. Arch Phys Med Rehabil. 1998;79(9):1101-4.

12. Ozdemir H, Soyuncu Y, Ozgorgen M et al. Effects of changes in heel fat pad thickness and elasticity on heel pain. J Am Podiatr Med Assoc. 2004; 94(1):47-52.

13. Wearing SC, Smeathers JE, Yates B et al. Bulk compressive properties of the heel fat pad during walking: A pilot investigation in plantar heel pain. Clin Biomech (Bristol, Avon). 2009;24(4):397-402.

14. Nolan D, Kennedy N. Effects of low-dye taping on plantar pressure pre and post exercise: An exploratory study. BMC Musculoskelet Disord. 2009;10:40.
15. Hyland MR, Webber-Gaffney A, Cohen L et al. Randomized controlled trial of calcaneal taping, sham taping, and plantar fascia stretching for the short-term management of plantar heel pain. J Orthop Sports Phys Ther. 2006;36(6):364-71.

16. Choi HJ, Nam KW. The Effect of Horseback Riding Simulator on Static Balance of Cerebral Palsy. J Korean Soc Phys Ther. 2014;26(4):269-73.

17. Yang DJ, Park SK, Kang JI et al. Effects of Changes in Postural Alignment on Foot Pressure and Balance of Patients with Stroke. J Korean Soc Phys Ther. 2014;26(4):226-33.

18. Russo SJ, Chipchase LS. The effect of low-dye taping on peak plantar pressures of normal feet during gait. Aust J Physiother. 2001;47(4):239-44.

19. Maria C, Mark B. Therapeutic Taping for Musculoskeletal Conditions. Churchill Livingstone. 2010.

20. Katoh Y, Chao EY, Laughman RK et al. Biomechanical analysis of foot function during gait and clinical applications. Clin Orthop Relat Res. 1983(177):23-33

21. Sullivan J, Burns J, Adams R et al. Plantar heel pain and foot loading during normal walking. Gait Posture. 2015;41(2):688-93.

22. Wearing SC, Smeathers JE, Urry SR. The effect of plantar fasciitis on vertical foot-ground reaction force. Clin Orthop Relat Res. 2003(409):17585.

23. Vicenzino B, McPoil T, Buckland S. Plantar foot pressures after the augmented low dye taping technique. J Athl Train. 2007;42(3):374-80. 